\title{
CARACTERÍSTICAS CLÍNICAS E LABORATORIAIS À ADMISSÃO DE 483 CRIANÇAS E ADOLESCENTES COM REFLUXO VÉSICO-URETERAL PRIMÁRIO: ESTUDO RETROSPECTIVO
}

\author{
José Maria Penido Silva, José Silvério S. Diniz, Eduardo A. Oliveira*, Viviane S. P. Marino, \\ Mariana R. Pimenta, Carolina C. Matos, Samana B. Vieira \\ Trabalho realizado na Unidade de Nefrologia Pediátrica do Hospital das Clínicas e Departamento \\ de Pediatria da Faculdade de Medicina da Universidade Federal de Minas Gerais, Belo Horizonte, MG.
}

RESUMO - OBJeTIVos. Avaliar retrospectivamente as características clínicas/radiológicas dos pacientes admitidos com refluxo vésico-ureteral primário (RVU) entre 1969-1999.

Métodos. Pacientes com RVU primário foram submetidos a um protocolo de avaliação clínica, laboratorial e radiológica e acompanhados longitudinalmente. $\mathrm{Na}$ admissão, após controle da bacteriúria e do início da quimioprofilaxia, foram realizados avaliação laboratorial e estudo de imagens do trato urinário. Esta consistiu de uretrocistografia miccional, urografia excretora, cintilografia renal (DMSA) e ultra-sonografia. Para o presente estudo foram utilizados os dados obtidos na admissão.

Resultados. Foram admitidos nesse período 483 pacientes. Houve predomínio do sexo feminino $(70 \%)$ e da cor branca (70\%). A média de idade ao diagnóstico foi de 26 meses, sendo que $92,5 \%$ das crianças já haviam apresentado infecção urinária. Um grupo de 710 unidades renais foi analisado. A distribuição do grau de refluxo foi a seguinte: grau I (49; 7\%); II (254; 36\%); III (190; 26\%); IV (I6I; $23 \%$ ) e V (56; 8\%). Um total de 249 unidades renais apresentaram dano renal à admissão. A distribuição quanto à intensidade da lesão foi a seguinte: leve $(36 \%)$, moderada $(34 \%)$ e grave $(30 \%)$. Houve um risco significativo maior de lesão renal grave no sexo masculino (OR $=1,74$, IC $95 \%=1,2-2,5, p=0,002)$.

Conclusäo. 0 RVU predominou no sexo feminino e em sua maioria apresentou-se em graus leves a moderados. Contudo, houve um alto percentual de dano renal à admissão e uma tendência de maior morbidade para o sexo masculino.

Unitermos: Refluxo vésico-ureteral. Nefropatia do refluxo. Fatores de risco. Crianças. Adolescentes.

\section{INTRODUÇÃO}

O refluxo vésico-ureteral primário (RVU) é originado de uma anormalidade estrutural da junção ureterovesical, caracterizado por um segmento submucoso anormalmente curto do ureter, permitindo uma ascensão da urina da bexiga até os ureteres e os rins. Estima-se que ocorra em cerca de $1 \%$ da população pediátrica e em $30 \%$ a $40 \%$ das crianças com infecção urinárial-3.

O RVU causa uma significativa morbidade, principalmente devido às infecções urinárias de repetição. Além disso, é uma das principais causas de insuficiência renal e hipertensão arterial em crianças e adolescentes. Embora não tenha ainda sido demonstrada, de maneira inequívoca, uma relação causal entre refluxo,

$$
\begin{gathered}
\text { *Correspondência } \\
\text { Rua Patagônia, 515/701 } \\
\text { CEP: 30320-080 - Belo Horizonte - MG } \\
\text { E-mail: eduolive@medicina.ufmg.br } \\
\text { Fax: (031) 224-3088 }
\end{gathered}
$$

infecção urinária e dano ao parênquima renal, as evidências de estudos clínicos e experimentais têm demonstrado uma forte associação entre esses eventos. Aproximadamente $10 \%$ dos pacientes acometidos apresentam um mau prognóstico, em termos de função renal, a longo prazo ${ }^{4,5}$.

Nos últimos anos, um maior conhecimento do curso clínico do RVU tem permitido uma abordagem mais racional e eficaz desse problema. Uma diversidade de fatores tem contribuído para esses avanços, incluindo aprimoramentos tecnológicos na obtenção de imagens do trato urinário, diagnóstico neonatal do refluxo através da investigação da hidronefrose fetal e estudos prospectivos controlados que avaliam a melhor conduta para esses pacientes ${ }^{1,2}$. Além disso, os avanços no conhecimento de biologia molecular têm permitido um conhecimento mais preciso da etiopatogênese do refluxo!.

O presente estudo tem sua origem na análise retrospectiva de uma casuística de crianças portadoras de RVU admitidas entre 1969 e 1999 na Unidade de Nefrologia Pediátrica do HC-UFMG. Tem como principais objetivos descrever as características clínicas, laboratoriais e radiológicas obtidas na admissão desses pacientes e avaliar preliminarmente os principais fatores associados à presença de dano renal.

\section{Métodos}

Pacientes. Os dados foram obtidos retrospectivamente dos prontuários dos pacientes portadores de refluxo vésico-ureteral primário encaminhados à Unidade de Nefrologia Pediátrica - Hospital das Clínicas - UFMG entre 1969 a 1999. Os pacientes foram admitidos para investigação de infecção do trato urinário e outros sinais e sintomas tais como incontinência urinária, enurese e hidronefrose fetal. Para a análise foram excluídos os casos de refluxo secundário associados à válvula de uretra posterior, ureterocele, bexiga neurogênica, entre outros. Desses prontuários 
foram anotados: sexo, idade, pressão arterial, infecção urinária e níveis séricos de uréia e creatinina.

Após comprovação da ausência de bacteriúria, foram realizados os exames de imagem, incluindo ultra-sonografia do trato urinário (US) e uretrocistografia miccional (UCM). O refluxo vésico-ureteral foi classificado em cinco graus de acordo com o International Reflux Study Committé. Para a análise estatística, o refluxo também foi classificado em leve (graus l e II) e moderado/grave (III a V). Para avaliação do trato urinário superior, foram utilizados os seguintes exames: urografia excretora (UE), cintilografia estática por TC99m-DMSA (DMSA) e US. Dos 483 pacientes admitidos, 98\% foram submetidos a pelo menos um método de imagem para avaliação da presença de dano renal. Contudo, os três métodos não foram realizados uniformemente em todos os pacientes. O método mais freqüente foi o DMSA, realizado em 189 pacientes (40\%) na admissão, seguido de UE em 164 casos (35\%) e US em 119 pacientes (25\%). O dano renal foi classificado em leve (apenas cicatriz localizada), moderado (múltiplas cicatrizes) e grave (rim contraído) ${ }^{7}$.

O estudo foi aprovado pelo Comitê de Ética do Hospital das Clínicas da UFMG em fevereiro de 2002.

Análise estatística. Para a realização do estudo foi construído um banco de dados no programa Epi Info, versão $6^{8}$. Para a comparação de proporções foi utilizado o teste do qui-quadrado. Foi também utilizado o cálculo do odds ratio (OR) e do intervalo de confiança a 95\% (IC 95\%) para a comparação de risco entre os grupos.

\section{Resultados}

\section{Dados clínicos na admissão}

Um grupo de 483 pacientes com RVU foi admitido no período estudado, sendo 24 (5\%) entre 1969-1979, I3। (27\%) entre 1980-1989 e 328 (68\%) entre 1990-1999. Houve predominância do sexo feminino (70\%) e da cor branca (70\%). A maioria dos pacientes foi encaminhada devido à infecção urinária $(92,5 \%)$ e hidronefrose fetal (5\%). A média de idade ao diagnóstico foi de 26 meses e mediana de 19 meses (I - 168 meses). Na Figura I, pode ser observada a distribuição da idade quando do diagnóstico em relação ao sexo. Nota-se que o

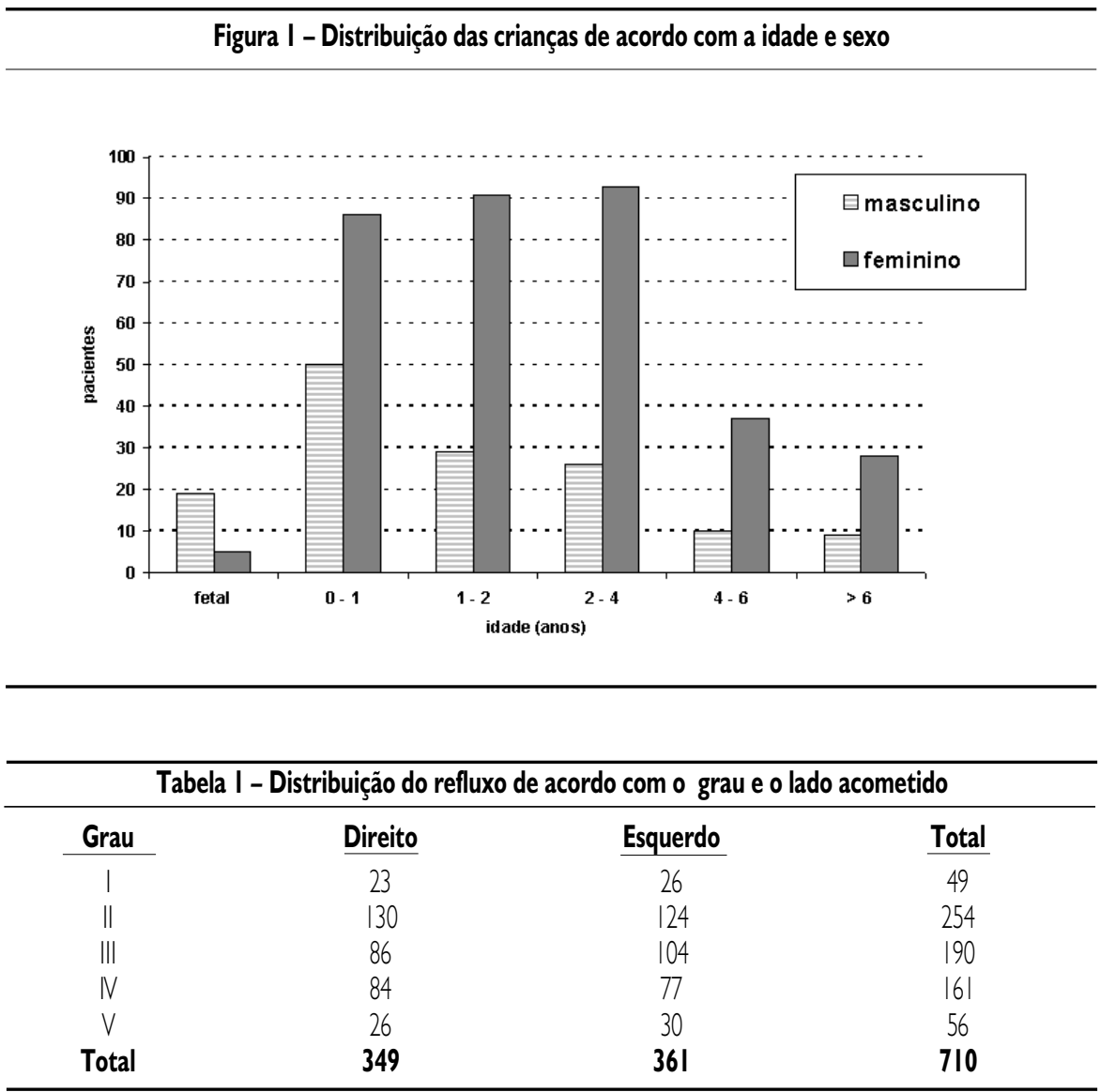

diagnóstico do RVU apresentou preponderância no sexo feminino em todas as faixas etárias, exceto no grupo diagnosticado na investigação de hidronefrose fetal. Até o primeiro ano de vida, o RVU já havia sido detectado em 47\% dos meninos e em apenas $27 \%$ das meninas. A mediana de idade do diagnóstico foi de 13 meses para os meninos e de 22 meses para as meninas $(p=0,03)$. Os casos de hidronefrose fetal foram responsáveis por essa diferença; excluindo-se esses casos, a mediana de idade do diagnóstico para os meninos seria de 28 meses e para as meninas de 31 meses $(p=0,34)$.

A pressão arterial foi avaliada em 401 pacientes à admissão. A pressão arterial sistólica encontrava-se acima do percentil 95 para a idade em 48 crianças (I2\%). A pressão arterial diastólica apresentou-se acima desse limiar em 36 pacientes (9\%). Os pacientes com dano renal grave apresentaram um risco três vezes maior de hipertensão arterial à admissão $(\mathrm{OR}=3,3, \mathrm{IC} 95 \%=1,5=7,5)$. A função renal foi obtida em apenas 280 pacientes na admissão. A média de uréia sérica foi $32 \mathrm{mg} / \mathrm{dl}$
(6 - 230), sendo que 45 pacientes apresentavam valores acima dos limites de referência. $A$ média da creatinina sérica foi de $0,67 \mathrm{mg} / \mathrm{dl}$ $(0,1-6,40)$, sendo que 30 pacientes apresentavam valores acima de $1 \mathrm{mg} / \mathrm{dl}$. O risco dos pacientes com dano renal grave bilateral apresentarem creatinina acima de I mg/dl foi cinco vezes maior $(O R=5, I C 95 \%=2-12)$.

\section{Dados do refluxo vésico-ureteral}

O diagnóstico do RVU foi obtido por uretrocistografia miccional em 46 I dos pacientes $(95 \%)$ e por cistografia radioisotópica direta em 22 casos (5\%). Dos 46 I submetidos a UCM, 249 apresentavam refluxo bilateral dando um total de 710 unidades para análise. A distribuição do grau de refluxo foi a seguinte: grau I (49 unidades; 7\%); grau II (254; 36\%); grau III (190; 26\%); grau IV (I6I; 23\%) e grau V (56; 8\%). Não houve diferença quanto à gravidade do refluxo quando comparados os lados direito e esquerdo $(p=0.6)$. A distribuição do refluxo quanto ao grau e o lado acometido pode ser observada na Tabela I. 
Os graus moderados a graves do refluxo predominaram nas crianças do sexo masculino. No sexo masculino, das 203 unidades com refluxo, 71\% apresentavam grau moderado a grave de refluxo; em contraste, no sexo feminino, das 507 unidades, apenas 52\% apresentavam essa distribuição $(O R=2,34$, IC $95 \%=1,6-3,4, p<0,001)$. A Figura 2 ilustra a distribuição do grau do refluxo de acordo com o sexo. Nota-se que graus elevados de refluxo predominaram no sexo masculino.

\section{Dano renal}

Durante todo o período estudado, houve uma alteração dos métodos utilizados na avaliação do trato urinário superior. Dos 472 pacientes avaliados, 152 foram admitidos até 1989. Desses, 104 (69\%) foram submetidos à UE, 3I (20\%) ao DMSA e 17 (I I\%) a US. Em contraste, dos 320 pacientes admitidos após 1990, os valores referentes a cada método foram 60 (19\%), I 58 (49\%) e 102 (32\%), respectivamente. Dos 472 (944 unidades renais) pacientes avaliados, 194 (41\%) apresentavam dano renal à admissão, sendo que em 55 casos a lesão foi bilateral. Assim, um total 249 (53\%) unidades renais apresentaram dano renal à admissão. A distribuição quanto à intensidade da lesão foi a seguinte: leve (89; $36 \%)$, moderada $(85 ; 34 \%)$ e grave $(75$; $30 \%)$. A presença e a intensidade de lesão renal estiveram associadas ao grau do refluxo. A Figura 3 ilustra a correlação entre o grau do refluxo e a ocorrência de cicatriz renal. Pode ser observado que, nas unidades sem refluxo ou com graus leves (I/II), mais de $90 \%$ não apresentavam dano renal. Em contraste, nas unidades com refluxo grau IVN, mais de $70 \%$ apresentavam lesão renal, em sua maioria de grau moderado a grave. A probabilidade das unidades renais com graus de refluxo moderado a grave (III a V) apresentarem dano renal foi significativamente maior. Das 365 unidades renais com grau moderado/grave de refluxo, 188 (52\%) apresentavam lesão renal. Das 293 unidades com graus leves $(I / I)$ de refluxo, esse percentual atingiu apenas $10 \%(O R=9,3, I C$ $95 \%=6-|4, p<0,00|)$.

Não houve correlação significativa entre a presença de lesão renal e o sexo do paciente, embora tenha havido uma maior tendência de risco de dano renal no sexo masculino. Das 284 unidades analisadas do sexo masculino, 86 (30\%) apresentaram dano renal na

Figura 2 - Correlação entre o sexo do paciente e o grau do refluxo na admissão

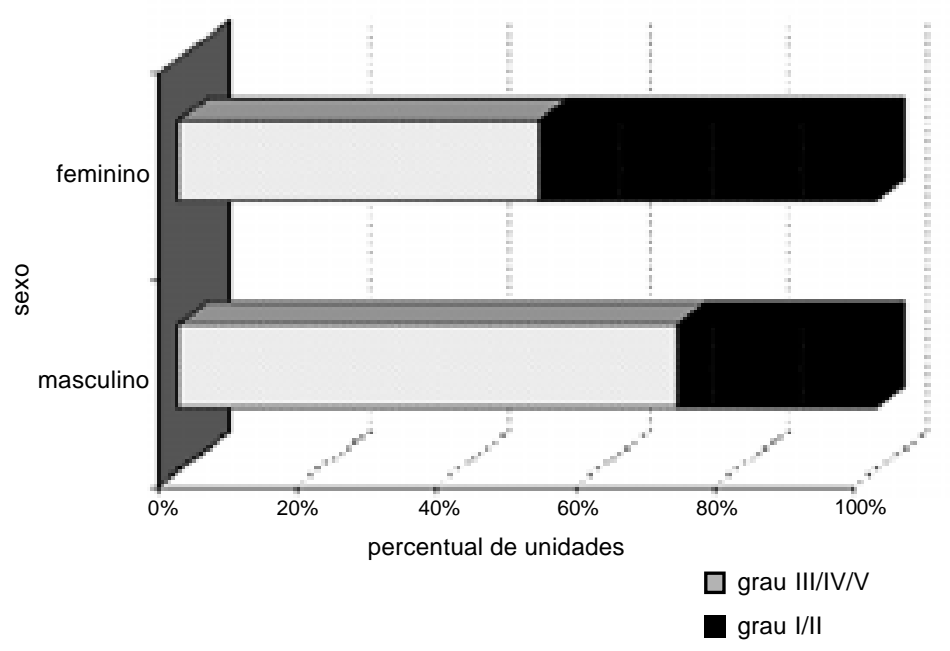

Figura 3 - Correlação entre o grau do refluxo e a presença e a gravidade do dano renal

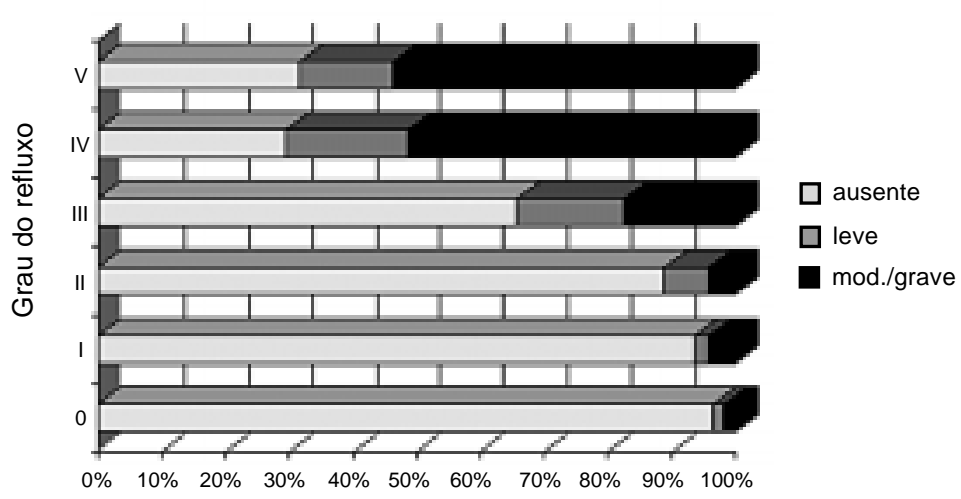

Unidades renais com dano renal

admissão; já das 660 unidades do sexo feminino, em 164 (25\%) foi demonstrada lesão renal $(\mathrm{OR}=1,31, \mathrm{IC} 95 \%=0,95-1,1, \mathrm{p}=0,09)$. Por outro lado, quando foi avaliada a presença de lesão renal moderada a grave, houve um risco significativo maior para o sexo masculino $(O R=1,74, I C 95 \%=1,2-2,5, p=0,002)$.

\section{Discussão}

O RVU e sua nefropatia associada têm sido objetos de intensos estudos clínicos e experimentais nas últimas três décadas. Nesse período, alguns avanços têm sido obtidos no reconhecimento da história natural da doença e na bem estabelecida tendência à resolução espontânea do refluxo. A abordagem do refluxo tem sido aprimorada, especialmente com a realização de ensaios clínicos com amostragem aleatória procurando discernir a meIhor opção terapêutica para o refluxo grave $e^{9-11}$. $O$ presente estudo relata dados provenientes de uma extensa série de crianças e adolescentes portadores de RVU admitidos em um 
único centro por aproximadamente 30 anos. Tratando-se de um estudo retrospectivo, envolvendo uma observação por tão longo tempo, é inevitável que algumas condutas tenham sido modificadas ao longo do período de observação. Por exemplo, a avaliação do trato urinário superior através da cintilografia renal somente se tornou disponível após o final da década de 80 e, assim, houve um nítido aumento na sensibilidade de detecção do dano renal ${ }^{12,13}$. Mesmo com essas ressalvas, alguns dados do presente estudo podem contribuir para a identificação de grupos de risco para uma lesão renal mais grave e, assim, estabelecer uma abordagem mais eficaz.

Os dados clínicos de nossa série são semeIhantes a vários outros estudos descritivos nos quais houve também uma predominância do sexo feminino e de pacientes de cor branca. Skoog et al. ${ }^{14}$ relataram uma série de 545 crianças com RVU, sendo 467 (86\%) do sexo feminino e 494 (90\%) da cor banca. No International Reflux Study in Children (IRSC), dos 452 pacientes admitidos, apenas 20\% eram do sexo masculino ${ }^{15}$. Em nossa série, a média de idade do diagnóstico foi 36 meses, sendo esse achado também comparável com outras grandes séries de refluxo ${ }^{14,16}$. Houve, em nossa casuística, uma grande preponderância de diagnóstico obtido na investigação após infecção urinária, embora 5\% dos casos de nossa série já sejam constituídos de refluxo "fetal". Houve predomínio do sexo feminino em todas as faixas etárias, exceto no período neonatal. Esse é um achado que tem sido consistentemente observado nas séries de refluxo diagnosticado na investigação de hidronefrose fetal. Recentemente, compilamos os achados de II séries de refluxo fetal nas quais predominou o sexo masculino, sendo a relação aproximadamente de $5: 1^{17}$. Esse fato também foi observado por Yeung et al. ${ }^{18}$ na maior série publicada até o presente momento, na qual foram avaliados I 55 neonatos, sendo I I 7 (75\%) do sexo masculino. Várias hipóteses têm sido levantadas para explicar esses achados. O refluxo seria freqüentemente subestimado nos meninos devido à menor incidência de infecção urinária ${ }^{19}$. Alguns estudos sugerem presença de obstrução uretral transitória intraútero no sexo masculino ${ }^{20}$. Anderson and Rickwood ${ }^{7}$ sugeriram que uma alta pressão miccional intra-uterina poderia distorcer a junção ureterovesical, causando refluxo e dano renal. Essa hipótese é apoiada pelo achado no pós-natal de importante disfunção miccional em meninos com refluxo "fetal"21.

Houve uma tendência de diagnóstico mais precoce no sexo masculino, além de um predomínio de graus mais elevados de refluxo nesse grupo de crianças. Esse fato também tem sido observado em séries recentemente publicadas. Goldraich et al. ${ }^{22}$ relataram que o refluxo já havia sido detectado no final do primeiro ano de vida em $56 \%$ dos meninos e em apenas 19\% das meninas em uma série de 202 crianças com RVU.

Em nosso estudo foi detectado dano renal em $52 \%$ das unidades avaliadas, com predomínio de lesões leves a moderadas. Houve associação de dano renal com graus mais elevados de refluxo e os casos de lesão grave predominaram no sexo masculino. Esse achado também foi coincidente com o estudo de Goldraich et al. ${ }^{22}$, no qual foi encontrada uma significativa preponderância de nefropatia do refluxo grave no sexo masculino, independentemente do grau do refluxo. Yeung et al. ${ }^{18}$ demostraram que o dano renal grave foi seis vezes mais freqüente no sexo masculino em uma série de 155 crianças com RVU detectado na investigação de hidronefrose fetal. Lama et al. ${ }^{23}$ detectaram dano renal em $85 \%$ das unidades renais com refluxo "fetal", sendo que no sexo masculino $86 \%$ dos rins estavam afetados e no sexo feminino esse percentual atingiu $75 \%$. Assim, parece haver uma tendência de uma maior repercussão clínica no sexo masculino. Dados recentes do Registro Italiano de Insuficiência Renal Crônica na Infância mostram que os pacientes do sexo masculino com RVU são o grupo predominante de perda da função renal entre I a $\mid 5$ anos de idade ${ }^{23}$.

Em nosso estágio atual de conhecimento, não se pode afirmar que o refluxo e o dano renal associado são meramente expressões separadas de uma mesma anomalia do desenvolvimento ou se há uma relação de causa/ efeito entre essas identidades. Os estudos mais atuais demonstram que parece haver pelos menos dois quadros clínicos distintos de RVU. Um grupo de maior risco, predominantemente do sexo masculino, com graus elevados de refluxo, um alto percentual de lesão renal e freqüentemente com disfunção miccional associada. Esse grupo tem sido muitas vezes identificado na investigação da hidronefrose fetal. $O$ outro grupo seria constituído predominantemente de meninas, com diagnóstico mais tardio do refluxo de grau leve a moderado, infecções urinárias de repetição e presença de cicatrizes localizadas.

Os mecanismos adquiridos de lesão renal têm tido maior ênfase nos estudos da gênese da chamada nefropatia do refluxo. Contudo, recentemente alguns fatos têm chamado mais a atenção para a contribuição dos fatores embriogenéticos na etiopatogenia do dano renal. Apesar da gênese do dano renal associado ao refluxo não ser ainda bem compreendida, fatos recentes têm contribuído para o conhecimento dos mecanismos envolvidos no dano renal. O diagnóstico do RVU a partir da investigação da hidronefrose fetal tem contribuído para uma melhor compreensão da história natural dessa uropatia e de suas conseqüências, antes que fatores intervenientes, tais como infecção urinária, alterem o curso clínico do refluxo. Convincentes evidências de uma associação entre displasia renal congênita e a nefropatia do refluxo têm sido demonstradas ${ }^{24-26}$. Outro importante avanço tem sido relacionado aos estudos da genética e de biologia molecular. Recentemente, Feather et al. ${ }^{27}$ identificaram um locus sugestivo de RVU no cromossomo Ip I3, entre GATAI76COI e DISI653. Esse achado, acoplado aos recentes estudos utilizando cepas mutantes de ratos para os receptores da angiotensina tipo 2 (AT2), pode representar importantes passos no entendimento dessa complexa questão ${ }^{28,29}$.

Concluindo, inegavelmente houve um progresso no conhecimento do RVU na última década. Os estudos clínicos mais recentes, incluindo a presente série, parecem apontar para grupos distintos de morbidade do refluxo. Nossos resultados podem contribuir na identificação de um grupo de crianças e adolescentes com maior risco de dano renal já na admissão e, portanto, com risco de evolução com comprometimento da função renal e da pressão arterial. Contudo, uma ressalva importante deve ser mencionada em relação ao nosso estudo. Trata-se, como enfatizado, de um estudo retrospectivo com suas inerentes limitações. Em nossa série, pode ter havido subestimação do dano renal nos pacientes admitidos nas primeiras décadas do estudo devido à limitação dos métodos de imagem da época. Assim, deve ser ressaltado que somente estudos prospectivos poderão estabelecer, com segurança, diferentes grupos de riscos e, assim, identificar adequadamente aqueles pacientes que possam merecer abordagem individualizada e mais eficaz. 


\section{Agradecimentos}

O presente estudo recebeu apoio financeiro da Fundação de Amparo à Pesquisa do Estado de Minas Gerais (FAPEMIG). As alunas Mariana R. Pimenta e Samana B. Vieira foram bolsistas de iniciação científica do CNPq e a aluna Carolina C. Matos foi bolsista do programa PAD (Pró-Reitoria de Graduação-UFMG).

\section{Conflito de interesse: não há}

\section{SUMMARY}

\section{Baseline clinical features of 483 CHILDREN AND ADOLESCENTS WITH PRIMARY VESICOURETERAL REFLUX: A RETROSPECTIVE STUDY}

OBJECTIVES, To evaluate the clinicall radiological features of patients with primary vesicoureteral reflux (VUR) admitted to a single institution from 1969 to 1999.

MetHODS. At admission, after the institution of chemoprophylaxis, patients were investigated by CUM, DMSA, and US. The children were managed with periodical clinical and laboratory evaluations. Analyses were performed with the data obtained at admission.

RESULTS. A total of 483 patients were enrolled in the protocol. There was a predominance offemales (70\%) and caucasian race (70\%). Mean age at VUR diagnosis was 26 months and $92.5 \%$ of the patients had urinary infection before admission. A total of 710 refluxing units were analyzed. The distribution of reflux grade was: grade I (49; 7\%); II (254; 36\%); III (190; 26\%); IV (I6I; 23\%) e $V(56 ; 8 \%)$. Approximately one half of the units analyzed were from patients presenting renal damage at admission. The distribution of the severity of renal damage was as follows: mild (36\%), moderate (34\%), and severe (30\%). There was a significant risk of severe renal damage for the males $(O R=1.74,95 \% \mathrm{Cl}=$ $1.2-2.5, p=0.002)$.

CONCLUSION. There was a predominance of VUR among females and most presented a mild or moderate degree. However, there was a high percentage of renal damage at admission and a tendency to greater morbidity for the males. [Rev Assoc Med Bras 2004; 50(2): 153-7]

Key words: Vesicoureteral reflux. Reflux nephropathy. Risk factors. Children. Adolescents.

\section{REFERÊNCIAS}

I. Belman AB. A perspective on vesicoureteral reflux. Urol Clin North Am 1995; 22:139-50.

2. Belman AB. Vesicoureteral reflux. Pediatr Clin North Am 1997; 44: I I I -90.

3. Smellie JM. Vesico-ureteric reflux. Acta Paediatr 1999; 88: I|82-3.

4. Bailey RR. The pathogenesis of vesicoureteric reflux and reflux nephropathy - what are we learning from antenatal ultrasonography? N Z Med J 1992; 105:423.

5. Dillon MJ, Goonasekera CD. Reflux nephropathy. J Am Soc Nephrol 1998; 9:2377-83

6. Lebowitz RL, Olbing H, Parkkulainen KV, Smellie JM, Tamminen-Mobius TE. International system of radiographic grading of vesicoureteric reflux. International Reflux Study in Children. Pediatr Radiol 1985; 15:105-9.

7. Anderson PA, Rickwood AM. Features of primary vesicoureteric reflux detected by prenatal sonography. $\mathrm{Br} J$ Urol 1991; 67:267-71.

8. Dean AG, Dean JA, Coulombier D. Epi Info, Version 6: a word processing, database, and statistics program for epidemiology on microcomputers. Atlanta: Centers for Disease Control and Prevention; 1994.

9. Prospective trial of operative versus nonoperative treatment of severe vesicoureteric reflux: two years' observation in 96 children. Br Med J 1983; 287: 171-4.

10. Weiss R, Duckett J, Spitzer A. Results of a randomized clinical trial of medical versus surgical management of infants and children with grades III and IV primary vesicoureteral reflux (United States). The International Reflux Study in Children. J Urol 1992; | 48: | 667-73.

I I. Smellie JM, Barratt TM, Chantler C, Gordon I, Prescod NP, Ransley PG, Woolf AS. Medical versus surgical treatment in children with severe bilateral vesicoureteric reflux and bilateral nephropathy: a randomised trial. Lancet 200 I; 357: 1329-33.

12. Goldraich NP, Ramos OL, Goldraich $I H$. Urography versus DMSA scan in children with vesicoureteric reflux. Pediatr Nephrol 1989; 3:I-5.

13. Goonasekera CD, Gordon I, Dillon MJ. I5year follow-up of reflux nephropathy by imaging. Clin Nephrol 1998; 50:224-31.

14. Skoog SJ, Belman AB, Majd M. A nonsurgical approach to the management of primary vesicoureteral reflux. J Urol 1987; 138:94 |-6.

15. Weiss R, Tamminen-Mobius T, Koskimies $O$, Olbing $H$, Smellie JM, Hirche $H$, et al. Characteristics at entry of children with severe primary vesicoureteral reflux recruited for a multicenter, international therapeutic trial comparing medical and surgical management. The International Reflux Study in Children. J Urol 1992; 148: 1644-9.
16. Ozen HA, Whitaker RH. Does the severity of presentation in children with vesicoureteric reflux relate to the severity of the disease or the need for operation? Br J Urol 1987; 60:1 10-2

17. Oliveira EA, Diniz JS, Silva JM, Rabelo EA, Pontes AK, Souza MF. Features of primary vesicoureteric reflux detected by investigation of fetal hydronephrosis. Int Urol Nephrol 1998; 30:535-41.

18. Yeung CK, Godley ML, Dhillon HK, Gordon I, Duffy PG, Ransley PG. The characteristics of primary vesico-ureteric reflux in male and female infants with pre-natal hydronephrosis. Br J Urol 1997; 80:319-27.

19. Scott JE. Fetal ureteric reflux. Br J Urol I987; 59:291-6

20. Avni EF, Schulman CC. The origin of vesicoureteric reflux in male newborns: further evidence in favour of a transient fetal urethral obstruction. Br J Urol 1996; 78:454-59.

21. Sillen U. Bladder dysfunction in children with vesico-ureteric reflux. Acta Paediatr Suppl 1999; 88:40-7

22. Goldraich NP, Goldraich IH. Followup of conservatively treated children with high and low grade vesicoureteral reflux: a prospective study. J Urol 1992; 148: 1688-92.

23. Lama G, Russo M, De Rosa E, Mansi L, Piscitelli A, Luongo I, et al. Primary vesicoureteric reflux and renal damage in the first year of life. Pediatr Nephrol 2000; 1 5:20510.

24. Stecker JF Jr, Rose JG, Gillenwater JY. Dysplastic kidneys associated with vesicoureteral reflux. J Urol 1973; I 10:341-3.

25. Hinchliffe SA, Chan YF, Jones $H$, Chan N, Kreczy A, Van Velzen D. Renal hypoplasia and postnatally acquired cortical loss in children with vesicoureteral reflux. Pediatr Nephrol 1992; 6:439-44.

26. Risdon RA, Yeung CK, Ransley PG. Reflux nephropathy in children submitted to unilateral nephrectomy: a clinicopathological study. Clin Nephrol 1993; 40:308-14.

27. Feather SA, Malcolm S, Woolf AS, Wright $V$, Blaydon D, Reid CJ, et al. Primary, nonsyndromic vesicoureteric reflux and its nephropathy is genetically heterogeneous, with a locus on chromosome I. Am J Hum Genet 2000; 66: 1420-5.

28. Woolf AS, Beales PL, Feather SA. Human malformation syndromes: lessons in kidney differentiation. Adv Nephrol Necker Hosp 1999; 29:329-39.

29. Woolf AS. A molecular and genetic view of human renal and urinary tract malformations. Kidney Int 2000; 58:500- 12. 